Supporting Information for

\title{
Distinct Cation-Anion Interactions in the UCST and LCST Behavior of Polyelectrolyte Complex Aqueous Solutions
}

Zhangxin Ye, ${ }^{a}$ Shengtong Sun, ${ }^{* b}$ and Peiyi $W{ }^{*}{ }^{* a b}$

aState Key Laboratory of Molecular Engineering of Polymers, Department of Macromolecular Science \& Laboratory for Advanced Materials, Fudan University, Shanghai 200433, China.

bState Key Laboratory for Modification of Chemical Fibers and Polymer Materials, College of Chemistry, Chemical Engineering and Biotechnology \& Center for Advanced Low-dimension Materials, Donghua University, Shanghai 201620, China.

Corresponding Author:

E-mail: shengtongsun@dhu.edu.cn

E-mail: wupeiyi@dhu.edu.cn 


\section{EXPERIMENTAL SECTION}

Materials. Poly(4-styrenesulfonic acid sodium salt) (PSSNa, Sigma-Aldrich, $M_{\mathrm{w}} \approx 500$ $\mathrm{kg} / \mathrm{mol}$ ), poly(diallyldimethylammonium chloride) (PDADMA-Cl, $20 \mathrm{wt} \%$ in water, $\left.M_{\mathrm{w}} \approx 150 \mathrm{~kg} / \mathrm{mol}\right)$ and potassium bromide $(\mathrm{KBr})$ were used as received. The homopolymer solutions of PSSNa and PDADMA-Cl were prepared at $50 \mathrm{mM}$ concentration with respect to their repeat units. PECs were prepared by mixing stoichiometric amounts of PSSNa and PDADMA-Cl solutions under stirring. The precipitate PECs were collected, pressed at 6000 psi pressure using Carver Press, and quickly rinsed with copious water. After being dried in a convection oven at $70{ }^{\circ} \mathrm{C}$ overnight, the PECs were ground into fine powders. The powders were intensively dried in vacuum at $150^{\circ} \mathrm{C}$ for $6 \mathrm{~h}$ and sealed in a container for further characterizations. Then, PEC powders were dissolved in $2.15 \mathrm{M} \mathrm{KBr}$ solutions with fixed concentrations, $C_{\mathrm{pi}}=0.15 \mathrm{M}$ and $0.3 \mathrm{M}$, respectively. The component solutions were vortex mixed repeatedly and stored at $5{ }^{\circ} \mathrm{C}$ in sealed vials for $24 \mathrm{~h}$ before performing further measurements.

Instruments and measurements. Turbidity measurements were carried on a UV-3150 spectrophotometer (Shimadzu, Japan) with a thermostat sample holder with a heating rate of $0.5^{\circ} \mathrm{C} / \mathrm{min}$. Optical microscopic observations were carried out on a Leica DM2500P polarizing microscope equipped with a Linkam THMS600 hot stage, whose heating rate was controlled manually at ca. $0.5^{\circ} \mathrm{C} / \mathrm{min}$. Raman spectra of PEC solutions were recorded on a Renishaw In Via Qontor/NTEGRA Spectra II spectrometer with the $532 \mathrm{~nm}$ laser (100\% power, $50 \mathrm{~mW}$; exposure time, $10 \mathrm{~s})$ as a light source. To increase temperature accurately, a Linkam THMS600 hot stage was applied to control the rate of temperature change at $0.5{ }^{\circ} \mathrm{C} / \mathrm{min}$. Temperature-dependent Raman spectra were recorded from 3 to $20{ }^{\circ} \mathrm{C}$ for $0.15 \mathrm{M}$ PEC aqueous solution and from 25 to $55^{\circ} \mathrm{C}$ for 0.3 M PEC solution. After all spectra were collected, the baselines of original spectra were uniformly corrected. 


\section{Investigation methods.}

Second-derivative Raman spectroscopy. The second-derivative Raman spectral analysis of PEC solutions were operated in the software, Omnic, ver. 8.2. The temperature-dependent Raman spectra of PEC $\left(C_{\mathrm{pi}}=0.15 \mathrm{M}\right)$ from $3-20{ }^{\circ} \mathrm{C}$ and $\mathrm{PEC}$ $\left(C_{\mathrm{pi}}=0.3 \mathrm{M}\right)$ from 25 to $55^{\circ} \mathrm{C}$ in $2.15 \mathrm{M} \mathrm{KBr}$ solutions were used for performing second-derivative analysis, which were then plotted overlapped to demonstrate the peak shifting trends in Figure 3.

Perturbation correlation moving window (PCMW). The temperature-dependent Raman spectra recorded with an increment of $1{ }^{\circ} \mathrm{C}$ during heating were employed to perform PCMW analysis. According to the method provided by Morita, ${ }^{\mathrm{S} 1}$ raw data processing was carried out. Further correlation information was calculated by the software 2D Shige, ver. 1.3 (CShigeaki Morita, Kwansei Gakuin University, Japan, 2004-2005) with an appropriate window size $(2 \mathrm{~m}+1=11)$ to generate good quality PCMW spectra. Finally, the contour maps were plotted using Origin, ver. 8.3 with red colors indicating positive intensities while green colors the negative ones.

Two-dimensional correlation analysis (2Dcos). The temperature-dependent Raman spectra of PEC $\left(C_{\mathrm{pi}}=0.15 \mathrm{M}\right)$ from 7 to $11^{\circ} \mathrm{C}$ and $\operatorname{PEC}\left(C_{\mathrm{pi}}=0.3 \mathrm{M}\right)$ from 34 to $51{ }^{\circ} \mathrm{C}$ in $2.15 \mathrm{M} \mathrm{KBr}$ solutions were used for performing 2D correlation analysis. 2D correlation analysis was carried out using the same software, 2D Shige, and was further plotted into contour maps using Origin program ver. 8.3. Similarly, red colors indicate positive intensities and green colors are defined as negative ones. 


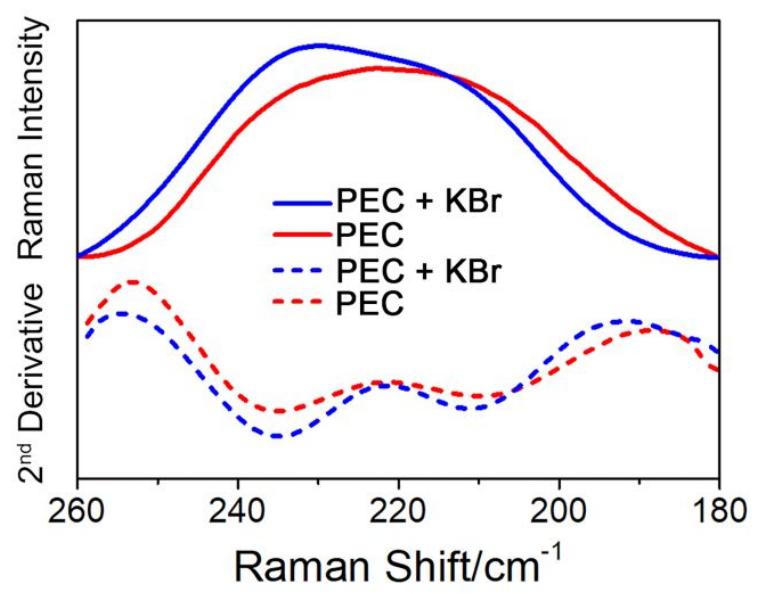

Figure S1. Contrast Raman spectra and the second-derivative spectra of $24 \mathrm{wt} \%$ watercontaining PECs with and without $\mathrm{KBr}$. Herein, the Raman intensities have been normalized for better comparison.
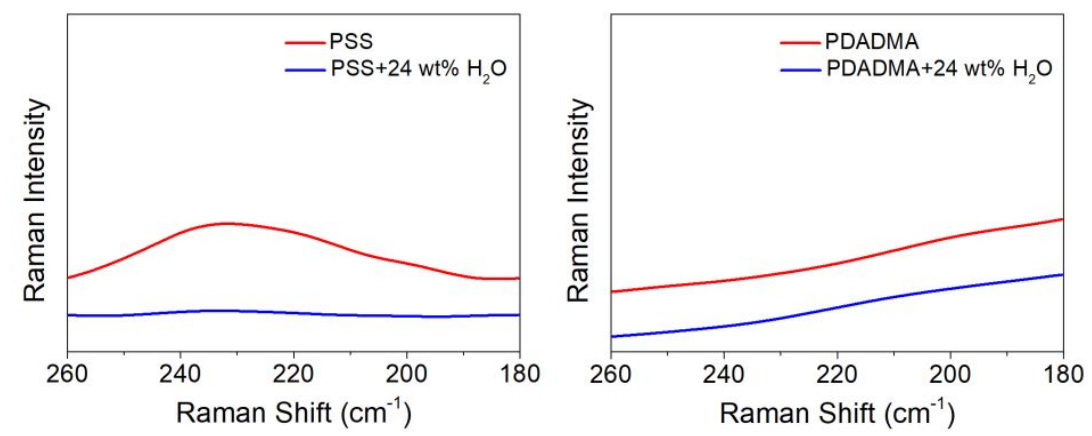

Figure S2. Contrast low-frequency Raman spectra $\left(180-260 \mathrm{~cm}^{-1}\right)$ of dried PSS or PDADMA films and hydrated PSS or PDADMA with 24 wt \% water. A peak at 234 $\mathrm{cm}^{-1}$ observed for dried PSS may come from salt-polymer interactions $\left(v\left(\mathrm{SO}_{3}{ }^{-\cdots} \mathrm{Na}^{+}\right)\right.$in the condensed solid phase, which is fully destroyed with the addition of water. No apparent Raman peaks are observed for dried PDADMA, and hydrated PSS and PDADMA. 

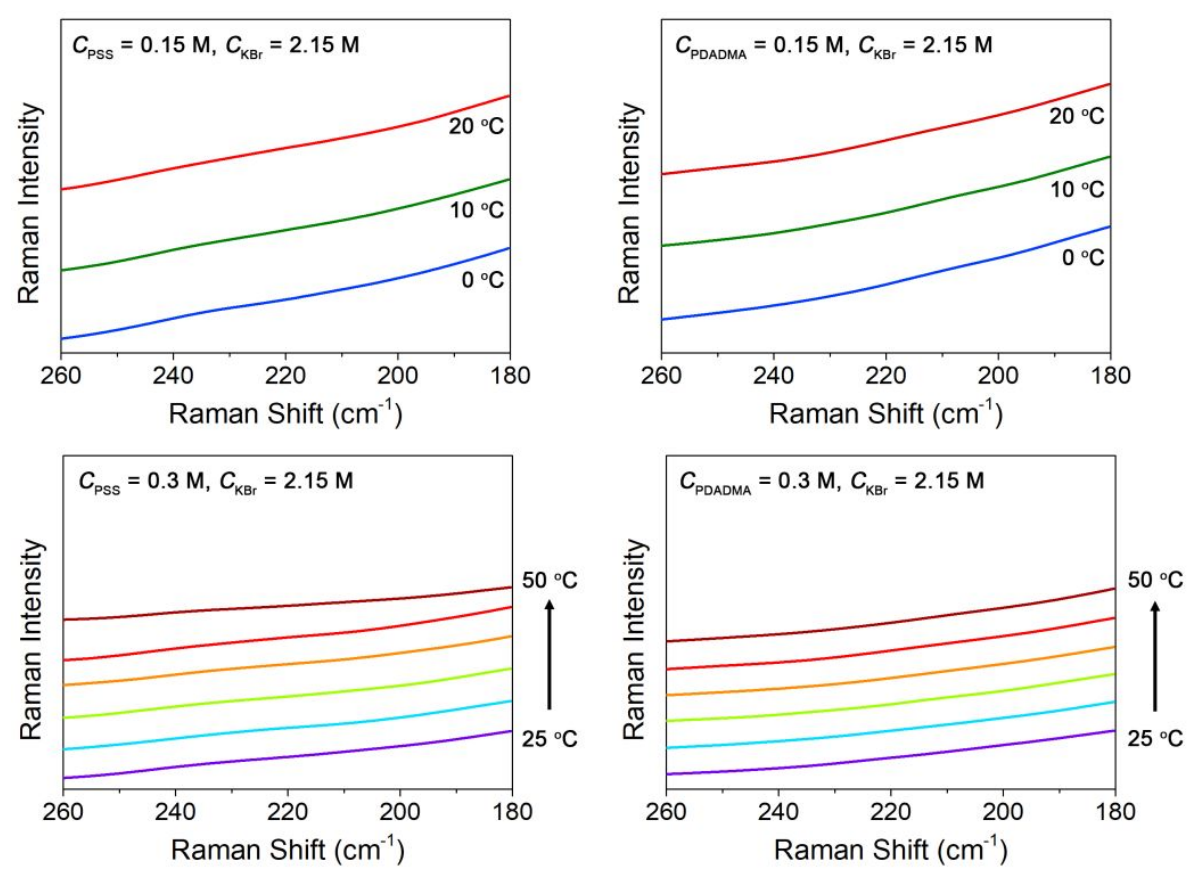

Figure S3. Temperature-dependent Raman spectra of PSS/KBr and PDADMA/KBr solutions with $C=0.15 \mathrm{M}, C_{\mathrm{KBr}}=2.15 \mathrm{M}$, from 0 to $20^{\circ} \mathrm{C}$ (typical for UCST), and $C$ $=0.3 \mathrm{M}, C_{\mathrm{KBr}}=2.15 \mathrm{M}$, from 25 to $50^{\circ} \mathrm{C}$ (typical for LCST), respectively. Temperature interval: $5{ }^{\circ} \mathrm{C}$. No recognizable peaks during the temperature-dependent Raman spectra of $\mathrm{PSS} / \mathrm{KBr}$ and $\mathrm{PDADMA} / \mathrm{KBr}$ solutions can be observed, confirming that the observed peak changes for the cation-anion interactions of PECs could only arise from polymer-polymer (PSS-PDADMA) interactions rather than salt-polymer interactions.

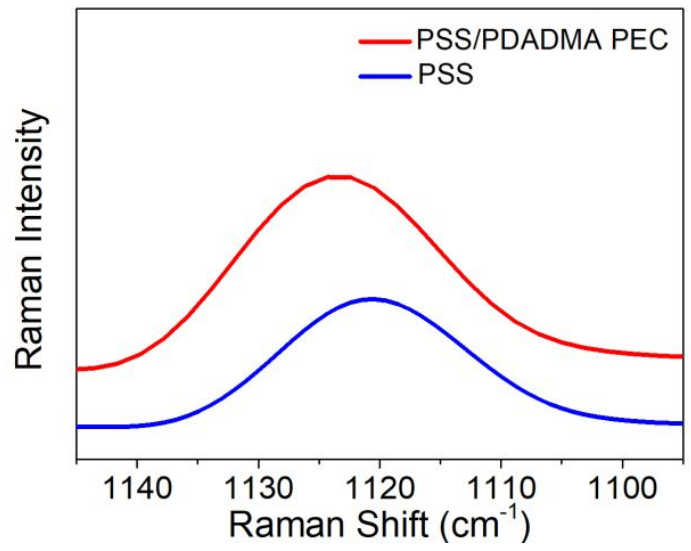

Figure S4. Contrast Raman spectra of $\mathrm{SO}_{3}{ }^{-}$groups in PSS and PSS/PDADMA PECs. The Raman shift of $v\left(\mathrm{SO}_{3}{ }^{-}\right)$of PEC is higher than that of free PSS. 

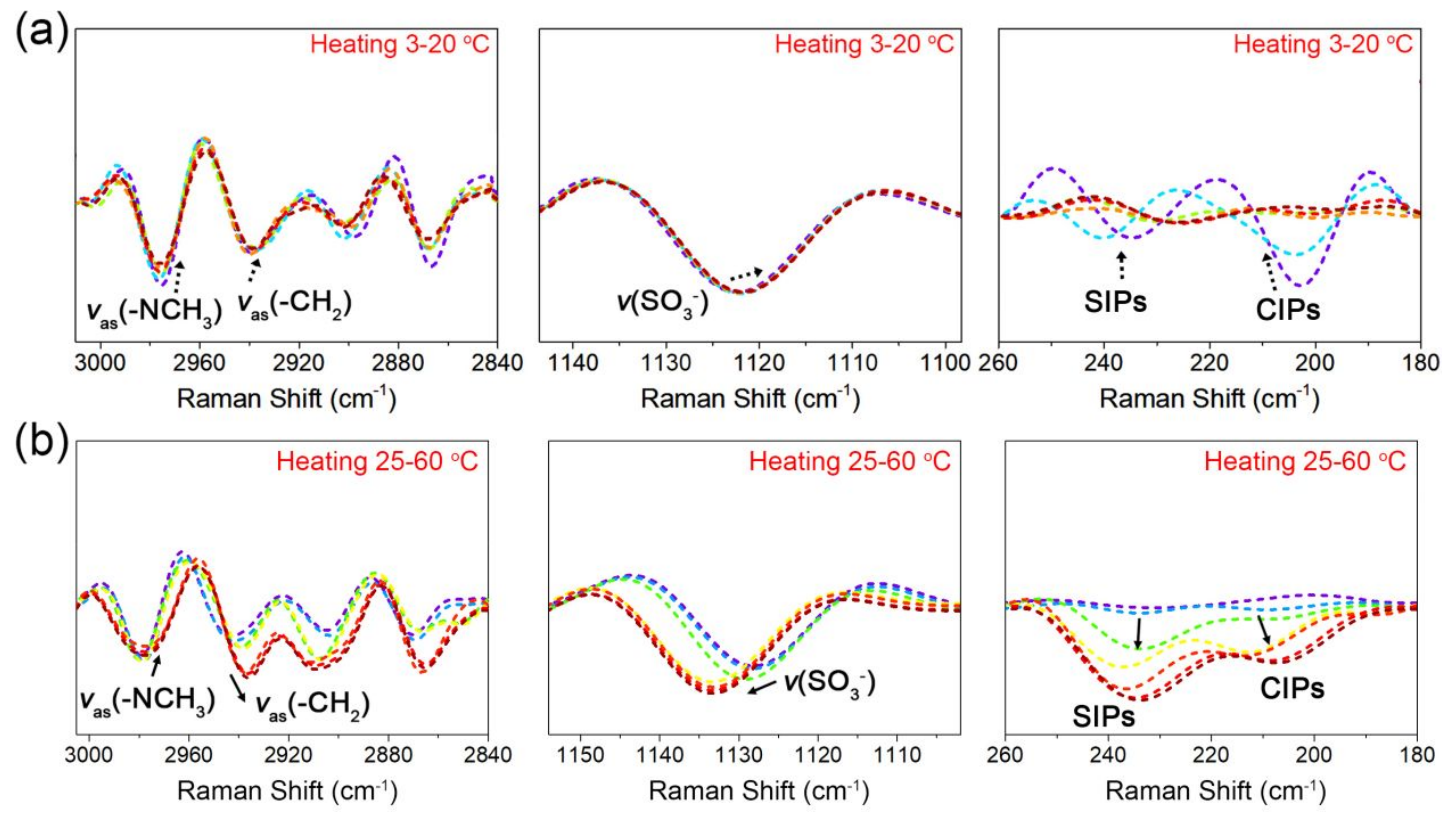

Figure S5. Temperature-dependent second-derivative Raman spectra of PEC solutions with (a) $C_{\mathrm{pi}}=0.15 \mathrm{M}, C_{\mathrm{KBr}}=2.15 \mathrm{M}$, from 3 to $20^{\circ} \mathrm{C}$, and (b) $C_{\mathrm{pi}}=0.3 \mathrm{M}, C_{\mathrm{KBr}}=2.15$ $\mathrm{M}$, from 25 to $55^{\circ} \mathrm{C}$. Second derivative spectroscopy is the technique of recording the second derivative of the spectrum with respect to wavelength/frequency, which offers great advantage over conventional spectroscopy if the absorbing species has sharp bands. Therefore, most of the spectral peaks or shoulder peaks that are not easily to be noticed in conventional 1D spectra can be identified in second-derivative spectra with minimum intensities and clearly readable peak positions. Here, the arrows as indicated in the above second-derivate Raman spectra consolidate all the observed variation trends in Figure 3.

\section{Perturbation Correlation Moving Window (PCMW)}

The PCMW method developed by Morita et al. was applied to monitor the sophisticated information on the accurate transition temperatures and transition temperature ranges. ${ }^{\text {S1-2 }}$ By means of PCMW, the conventional Raman spectra can offer a pair of synchronous and asynchronous correlations between spectral (e.g., Raman shift) and perturbation (e.g., temperature) variables. The positive synchronous correlation represents an increase in the spectral intensity, whereas the negative synchronous correlation indicates intensity decrease. With respect to the asynchronous correlation, 
the positive one denotes a convex spectral intensity variation and the negative one denotes a concave intensity variation. The temperature to which the strongest spectral intensity at the specific wavenumber in the synchronous and asynchronous spectra can be utilized to determine the transition temperature and transition temperature region of a certain chemical group. As shown in Figure S6, the UCSTs of most groups are ca. 9 ${ }^{\circ} \mathrm{C}$ with a transition temperature region of $7-11{ }^{\circ} \mathrm{C}\left(C_{\mathrm{pi}}=0.15 \mathrm{M}\right)$, while the LCSTs of most groups are consistent at $43{ }^{\circ} \mathrm{C}$ with a transition region of $34-51{ }^{\circ} \mathrm{C}$.

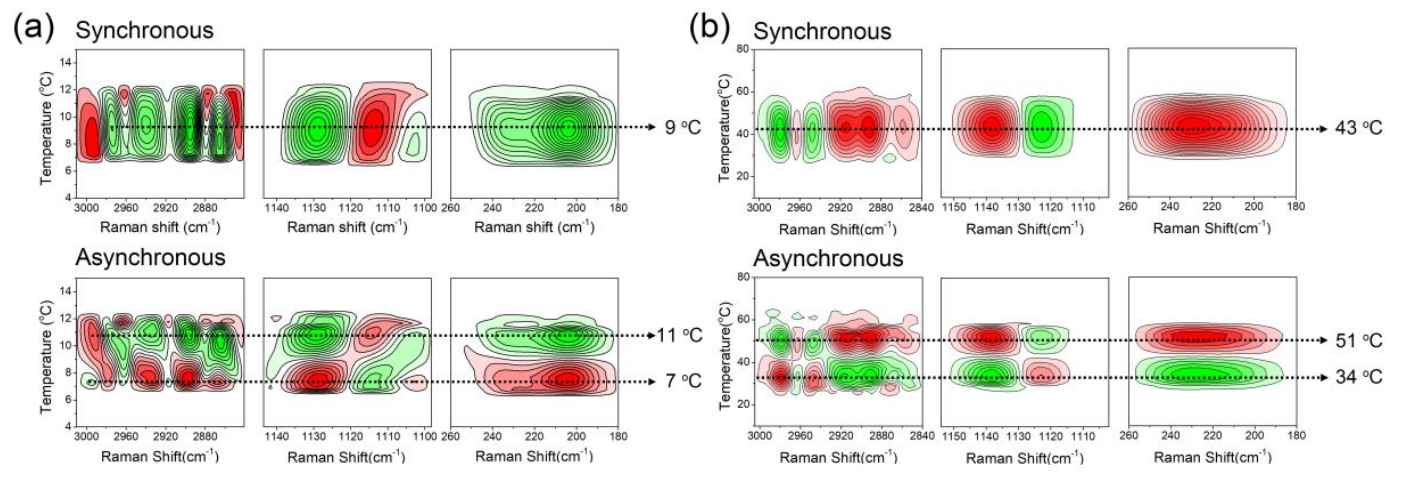

Figure S6. PCMW synchronous and asynchronous Raman spectra of PECs in $2.15 \mathrm{M}$ $\mathrm{KBr}$ aqueous solutions at the concentration of (a) $C_{\mathrm{pi}}=0.15 \mathrm{M}$ and (b) $C_{\mathrm{pi}}=0.3 \mathrm{M}$ during heating.

\section{Two-Dimensional Correlation Spectroscopy (2Dcos)}

2Dcos, as a mathematical method, is very suitable for the investigation of the variations of chemical groups. Its basic principle was first proposed by $\mathrm{Noda}^{\mathrm{S} 3-4}$ and have been applied more and more widely to follow spectral variations of different chemical groups under external perturbations, such as time, temperature, pressure, concentration, other physical variables, etc. By spreading the original spectra along a second dimension, spectral resolution enhancement can be achieved, allowing the additional important information about molecular motions or conformational changes not readily visible in conventional analysis to be extracted.

2Dcos includes two types of correlation maps, synchronous spectra which reflect simultaneous changes between two wavenumbers, and asynchronous spectra which can significantly enhance the spectral resolution. The auto-peaks only appear along the 
diagonal in synchronous spectra, while the cross-peaks can appear in both synchronous and asynchronous spectra. The judging rule of the sequence can be summarized as Noda's rule- that is, if the multiplication of the signs of cross-peaks $\left(v_{1}, v_{2}\right.$, and assume $v_{1}>v_{2}$ ) in synchronous and asynchronous spectra is positive, the change at $v_{1}$ may occur prior to $v_{2}$, and vice versa. ${ }^{\mathrm{S} 5-6}$ In the following tables, "+" means the same signs in synchronous and asynchronous spectra, while “_" means different signs in synchronous and asynchronous spectra. For instance, for $C_{\mathrm{pi}}=0.3 \mathrm{M}, C_{\mathrm{KBr}}=2.15 \mathrm{M}$, from 34 to 51 ${ }^{\circ} \mathrm{C}$ in Figure 5 (LCST case), in synchronous spectrum, the sign of the cross-peak (1141, 1132 ) is positive (red color); in asynchronous spectrum, the sign of such cross-peak is negative (green color). Therefore, the multiplication of the both signs for the crosspeaks $(1141,1132)$ is “-”, suggesting that the response of the species at $1132 \mathrm{~cm}^{-1}$ is prior to that at $1141 \mathrm{~cm}^{-1}$, or $v\left(\mathrm{SO}_{3}{ }^{-\cdots} \mathrm{H}-\mathrm{O}-\mathrm{H}^{\cdots+} \mathrm{N}\right)>v\left(\mathrm{SO}_{3}{ }^{-\cdots+} \mathrm{N}\right)$.

Table S1. Tentative band assignments of PSS/PDADMA PECs in the cases of UCST and LCST from 2Dcos.

\begin{tabular}{lll}
\hline Raman shift $\left(\mathrm{cm}^{-1}\right)$ & & Tentative assignments \\
\hline UCST & LCST & \\
\hline 1130 & 1141 & $v\left(\mathrm{SO}_{3}{ }^{-\cdots+} \mathrm{N}\right)$ \\
1122 & 1132 & $v\left(\mathrm{SO}_{3} \cdots+\mathrm{H}-\mathrm{O}-\mathrm{H}^{\cdots+} \mathrm{N}\right)$ \\
1114,1105 & 1121,1111 & $v\left(\mathrm{SO}_{3} \cdots \mathrm{H}_{2} \mathrm{O}\right)$ \\
225 & & $\mathrm{SIPs}$ \\
203 & 229 & $\mathrm{CIPs}$ \\
\hline
\end{tabular}


Table S2. Final results of multiplication of the signs of each cross-peak in synchronous and asynchronous spectra of PEC $\left(\mathrm{C}_{\mathrm{pi}}=0.15 \mathrm{M}\right)$ in $2.15 \mathrm{M} \mathrm{KBr}$ aqueous solution from 7 to $11^{\circ} \mathrm{C}(\mathrm{UCST})$.

\begin{tabular}{|c|c|c|c|c|c|c|}
\hline 1130 & & & & & & \\
\hline 1122 & + & & & & & \\
\hline 1114 & + & + & & & & \\
\hline 1105 & + & + & - & & & \\
\hline 225 & - & - & - & - & & \\
\hline \multirow[t]{2}{*}{203} & - & - & - & - & - & \\
\hline & 1130 & 1122 & 1114 & 1105 & 225 & 203 \\
\hline
\end{tabular}

The final specific order for UCST-type phase transition of PEC is given as follows: 225 $>203>1130>1122>1105>1114 \mathrm{~cm}^{-1}$, or $\mathrm{CIPs}>\mathrm{SIPs}>v\left(\mathrm{SO}_{3}{ }^{-\cdots+} \mathrm{N}\right)>v\left(\mathrm{SO}_{3}{ }^{-\cdots} \mathrm{H}_{-}\right.$ $\left.\mathrm{O}-\mathrm{H}{ }^{\cdots+} \mathrm{N}\right)>v\left(\mathrm{SO}_{3}{ }^{-\cdots} \mathrm{H}_{2} \mathrm{O}\right)$.

Table S3. Final results of multiplication of the signs of each cross-peak in synchronous and asynchronous spectra of $\mathrm{PEC}\left(\mathrm{C}_{\mathrm{pi}}=0.3 \mathrm{M}\right)$ in $2.15 \mathrm{M} \mathrm{KBr}$ aqueous solution from 34 to $51^{\circ} \mathrm{C}(\mathrm{LCST})$.

\begin{tabular}{|c|c|c|c|c|c|c|}
\hline 1141 & & & & & & \\
\hline 1132 & - & & & & & \\
\hline 1122 & - & + & & & & \\
\hline 1111 & - & - & - & & & \\
\hline 229 & - & - & - & - & & \\
\hline \multirow[t]{2}{*}{216} & - & - & - & - & + & \\
\hline & 1141 & 1132 & 1122 & 1111 & 229 & 216 \\
\hline
\end{tabular}

The final specific order for LCST-type phase transition of PEC is given as follows: 216 $>229>1111>1132>1122>1141 \mathrm{~cm}^{-1}$, or SIPs $>\mathrm{CIPs}>v\left(\mathrm{SO}_{3}{ }^{-\cdots} \mathrm{H}_{2} \mathrm{O}\right)>v\left(\mathrm{SO}_{3} \cdots \mathrm{H}-\right.$ $\left.\mathrm{O}-\mathrm{H}{ }^{\cdots+} \mathrm{N}\right)>v\left(\mathrm{SO}_{3}{ }^{-\cdots+} \mathrm{N}\right)$. 


\section{REFERENCES}

S1. Morita, S.; Shinzawa, H.; Noda, I.; Ozaki, Y., Perturbation-Correlation MovingWindow Two-Dimensional Correlation Spectroscopy. Appl. Spectrosc. 2006, 60 (4), $398-406$.

S2. Watanabe, A.; Morita, S.; Ozaki, Y., Study on Temperature-Dependent Changes in Hydrogen Bonds in Cellulose I $\beta$ by Infrared Spectroscopy with PerturbationCorrelation Moving-Window Two-Dimensional Correlation Spectroscopy. Biomacromolecules 2006, 7 (11), 3164-3170.

S3. Noda, I., Two-Dimensional Infrared Spectroscopy. J. Am. Chem. Soc. 1989, 111 (21), 8116-8118.

S4. Noda, I., Two-Dimensional Infrared (2D IR) Spectroscopy: Theory and Applications. Appl. Spectrosc. 1990, 44 (4), 550-561.

S5. Noda I, Ozaki Y. Two-Dimensional Correlation Spectroscopy: Applications in Vibrational and Optical Spectroscopy. Chichester: Wiley, 2004.

S6. Sun, S.; Wu, P. Spectral Insights into Microdynamics of Thermoresponsive Polymers from the Perspective of Two-Dimensional Correlation Spectroscopy. Chin. J. Polym. Sci. 2017, 35, 700-712. 